\title{
Impact of Telemedicine Tools on Record Keeping and Compliance in Haemophilia Care
}
A. Banchev ${ }^{1,2, *}$
G. Goldmann ${ }^{1, *}$
N. Marquardt ${ }^{1}$
C. Klein ${ }^{1}$
S. Horneff ${ }^{1}$
R. Langenkamp ${ }^{1}$
T. Frankenberger ${ }^{1}$ J. Oldenburg ${ }^{1}$
${ }^{1}$ Institute for Experimental Haematology and Transfusion Medicine,
University Hospital Bonn, University of Bonn, Bonn, Germany
2 Department of Paediatric Haematology and Oncology, University
Hospital “Queen Giovanna - ISUL," Sofia, Bulgaria
Address for correspondence Atanas Banchev, MD, Department of Paediatric Haematology and Oncology, University Hospital Queen Giovanna - ISUL, UI. Byalo More 8, 1527 Sofia, Bulgaria (e-mail: a.banchev@sbaldohz.com).

Hämostaseologie 2019;39:347-354.

\begin{abstract}
Keywords

- e-diary

- paper diary

- Haemoassist

- compliance

Background Record keeping is integral to home treatment for haemophilia. Issues with paper diaries include questionable compliance, data validity and quality. Implementation of electronic diaries (e-diaries) in haemophilia patients could improve documentation of home treatment.

Aim This article evaluates the effects of an e-diary, Haemoassist, on recording and patient compliance with therapy.

Patients and Methods An explorative study was used to assess the sequential use of paper diaries and e-diaries by 99 patients with severe haemophilia A or B and 1 with severe factor VII deficiency. Median age was 41 years. Information was obtained from paper records for 3 years preceding the introduction of an electronic record system and the first 6 to 12 months of Haemoassist use. Data from the 3-year period were averaged. Missing data for rounded 12 months of e-diary use were extrapolated to correspond to a full year.

Results Enhancement of $23 \%$ in record delivery was observed for the period of Haemoassist use ( $p=0.013)$. Twenty-one percent increase in patients' compliance for data reporting (from $65 \% 35$ to $86 \% 22, p=0.003$ ) and $16 \%$ increase for documentation of bleedings (from 68 to $84 \%$ of patients, $p=0.01$ ) were detected. Compliance to prescribed therapy of patients for the whole studied period improved by $6 \%$ (from $82 \% \pm 29$ to $88 \% \pm 25, p=0.05$ ). Major advances were demonstrated predominantly in the age groups of between 13 and 20 and 21 and 40 years.

Conclusion e-Diaries' use enables improved recording of information about patients' home treatment and bleeding episodes. Enhanced compliance with therapy may be a further benefit.
\end{abstract}

\section{Introduction}

The introduction of home-based therapy controlled by a medical doctor, which allowed the implementation of satisfactory prophylactic treatment regimens, led to significant improvements in haemophilia care over the past 30 years,

\footnotetext{
* A.B. and G.G contributed equally to this work.
}

received

March 31, 2018

accepted after revision

October 31, 2018

foremost in the western countries. One of the most significant advances is the decreased risk of death from bleeding and its complications ${ }^{1,2}$ as well as the improvement in the quality of life for people with haemophilia $(\mathrm{PwH})$-such as prevention of bleeds, reduction of joint damage ${ }^{3}$ and minimization of hospital stay. ${ }^{4}$ In general, home-based therapy grants autonomy and flexibility in patients' daily life. However, the documentation of concentrate infusions and home therapy tracking remains an

(c) 2019 Georg Thieme Verlag KG Stuttgart · New York
DOI https://doi.org/ 10.1055/s-0038-1676128. ISSN 0720-9355.

October 31,2018 
open issue, as the majority of patients present themselves in comprehensive care centres (CCCs) only a few times a year and most of their factor infusions take place in the absence of a treating physician. Therefore, the accurate documentation is of great importance for medical professionals to monitor the incidence of bleeding episodes, factor usage and compliance with treatment regimens. According to the current regulations in Germany (Transfusion Law), ${ }^{5}$ each applied infusion, its indication, the amount of infused factor concentrate along with brand name and batch number of the utilized product must be documented into the so-called 'protocols of therapy' and later on submitted to the treatment center. ${ }^{6}$ Patients' paper diary is a widely accepted means of record documentation. Although easily assessable, paper diaries harbour numerous drawbacks. They only allow retrospective tracking of the applied treatment regimen. Sometimes, patients complete them shortly before the upcoming visit which makes the collection of accurate and meaningful data on bleeding episodes, treatment regimen, factor usage and compliance to therapy difficult. ${ }^{7}$ Many patients forget to bring their diaries during visits to the treatment centre or enter data in the diaries with unclear handwriting. Consequently, neglected reporting may lead to inappropriate medical care. ${ }^{4}$ Interestingly, studies show that patients find paper diaries cumbersome and inconvenient. ${ }^{8}$ Furthermore, statistical analysis of data exported from paper diaries is time-consuming as they must be manually transferred to the corresponding electronic database. ${ }^{9}$

Due to the technological development and introduction of telemedicine, ${ }^{10}$ besides paper diaries, an evolving option for CCC becomes the use of electronic diaries (e-diaries). ${ }^{11}$ Studies conducted among patients with chronic pain ${ }^{12}$ prove that its implementation led to increased patients' compliance with data entry. Other publications suggest that digital diaries increase the amount of reported information, allowing real-time entries and clotting factor trafficking. ${ }^{8}$ Direct data analysis is seen as a further advantage of e-diaries. Questionnaire-based studies showed high patients' satisfaction with e-diary use. ${ }^{8}$

We conducted a study aiming at evaluating the effects of an electronic smartphone application/online diary named 'Haemoassist' (developed and introduced by 'Pfizer'), on quality of data documentation and patients' compliance to prescribed home therapy.

\section{Patients}

A total of 100 patients with severe coagulation factor deficiencies who had been using the respective e-diary for a period of 6 to 12 months were recruited to the study. All of them had been followed for at least 3.5 years at our treatment centre. Ninety-nine patients had severe haemophilia $A$ or B, and 1 had severe factor VII deficiency. By the time of evaluation, the mean age of patients was 41 years (range: 478 years), 7 of them were below 13 years, 4 patients between 13 and 20 years, 38 patients between 21 and 40 years, 35 patients between 40 and 60 years and 16 were older than 60 years. For young children in our group, the parents documented the treatment in the e-diary.

\section{Study Design and Methods}

In this explorative single-centre study, we analysed the effect of an e-diary on the treatment of $\mathrm{PwH}$, based on two consecutive periods of reporting-3 years before and 6 to 12 months after the introduction of e-diary. All recruited patients agreed on using the e-diary. After the switch from paper diary, the use of Haemoassist remained the only option for record keeping.

\section{Data Collection}

Data on the 3-year period before the introduction of Haemoassist were derived from the electronic database of Haemophilia Centre in Bonn, Germany. This electronic system holds data from historical paper records transferred manually by the treatment centre staff into the electronic files of each patient. Data collection comprised the number of paper entries per month returned to the centre (so-called 'protocols of clotting factor tracking' required by the German Transfusion Law), the day, the amount and the indication/ reason for clotting factor application (prophylaxis and/or bleeding events) over a period of 3 years before the introduction of Haemoassist. The 3-year period data were averaged.

Comparable information was derived from the e-diary server, regarding the first 6 to 12 months of Haemoassist use for the same patients. For patients who have been using the application for less than rounded 12 months, missing information was extrapolated to correspond to a full year to ensure comparability of data from both analysed periods.

\section{Age Groups}

By means of statistical analysis, all patients were allocated to five age groups (below 13 years of age, 13-20 years, 21-40 years, 41-60 years, older than 60 years) based on age-dependent differences in the lifestyles and mode of treatment.

\section{Assessments}

The following parameters were evaluated within our study:

1. Assessment of the impact of the switch to e-diary on data delivery for clotting factor application. Evaluated was the number of patients with fully filled-in monthly 'protocols' returned to the centre by the time of paper diary use. Additionally, the number of patients with present or missing reports for clotting factor application since the beginning of Haemoassist use was assessed.

2. Assessment of the impact of the switch to e-diary on completeness of data reporting. This parameter is presented in percentages obtained from the actual number of clotting factor infusions, reported monthly by the patient, divided by the definite number of expected monthly infusions according to the prescribed regimen. Completeness of data reporting was considered $0 \%$ in case information for clotting factor application was missing from both types of diaries (patient had either not been returning any paper diaries [protocols] to the centre or had not been entering any data into the Haemoassist system). Data from the 3-year paper diary use are 
presented as means and corresponding standard deviations (SDs) of the averaged values for completeness for each of the 3 years of observation. Data from the period of e-diary use are presented as means and SD.

3. Assessment of the impact of the switch to e-diary on compliance with the prescribed regimen. Compliance to the prescribed treatment regimen was assessed only for those patients who have reported throughout the studied period (patients with missing information for any of the analysed months of the observational period were excluded from the analysis). Thus, the avoidance of possible confounding in the assessed parameter due to the lack of patients' reporting was ensured. The parameter is expressed in percentages, where $0 \%$ was considered in case the patient was infusing clotting factor, but never according to the physician's prescription. Value of $100 \%$ corresponds to infusions applied in full concordance with the weekly factor application regimen prescribed by the physician. Data for both periods of comparison are presented as means and SD.

4. Assessment of the impact of the switch to e-diary on reporting of bleeding episodes is evaluated by analysing the number of patients who were reporting on bleeding events by the time of paper diary use, compared with the number of patients entering data about bleeds into the e-diary system after its implementation. The presence of bleeding episodes, their absence or lack of reporting was validated after a parallel check-up of patients' files in the haemophilia centre and clotting factor consumption log. The 3-year period data were averaged.

All home applications of clotting factor were validated by verification of the amount of clotting factor concentrate, dispensed by the treatment centre to the patient for the same period.

The study did not take into consideration prior computer experience of patients enrolled, as they were randomly switched to the use of e-diary. On the day of the switch to digital diary, each subject (irrespective of age and social status) was briefly instructed about the use of the Haemoassist application/online version by one of the CCC physicians.

Statistical analysis, figures and diagrams were processed using Excel 2016 and R-commander in Excel. As the distribution of data proved to be skewed, non-parametric Wilcoxon signed-rank test was used to prove the significance of the difference for the compared results. $p$-Values of $<0.05$ were considered significant.

'Pfizer', the supplier of 'Haemoassist' did not provide financial support for the study and had no influence on its design. All patients included in the analysis were coded by means of ensuring confidentiality.

\section{Results}

\section{Assessment of the Impact on Data Delivery}

Analysis of data delivery to site showed that before the use of ediary 23 of 100 patients were not delivering any information about home therapy during the observed 3 years of paper diary use, 5 of 100 were providing full information for only 1 year and



Fig. 1 Percentage of patients, delivering records on infusions during the time of paper diary use.

5 of 100 were providing full data for 2 years of the studied period ( - Figs. 1 and 2). After the implementation of Haemoassist, only 10 of 100 patients were not entering any treatment data into the system. The distribution according to age groups as a cross-section for both periods is presented in percentages in - Fig. 3. Major improvement (34\% more patients) was seen in the group of 21 to 40 years, contrary to the group of adolescents (13-20 years) which remained without any assessed progress. Overall enhancement in data delivery of $23 \%(p=0.013)$ was observed among the whole cohort.

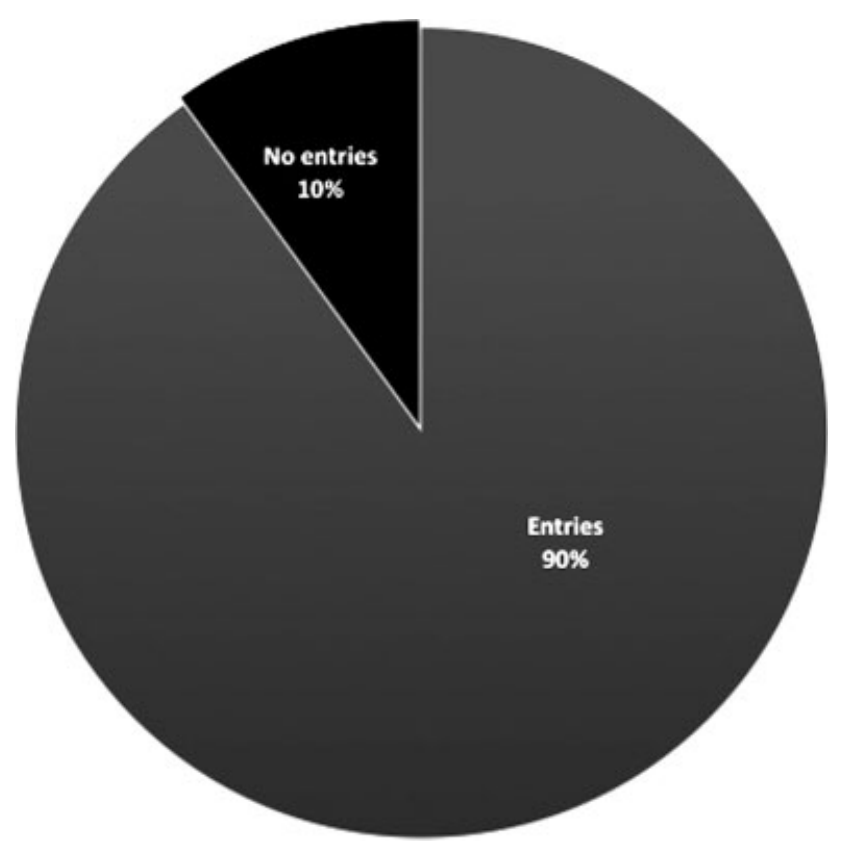

Fig. 2 Percentage of patients, delivering records on infusions during the time of e-diary use. 


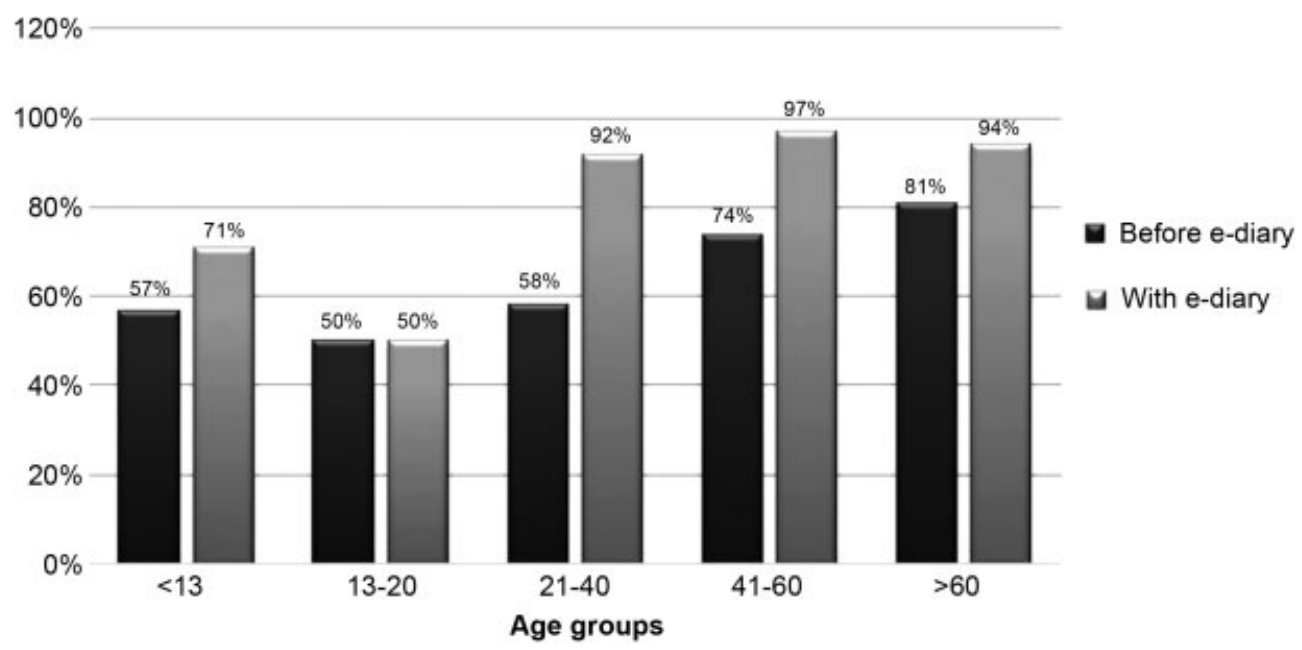

Fig. 3 Age distribution and comparison of record delivery for the period of paper diary use and e-diary use.

\section{Assessment of the Impact on Completeness of Data Reporting}

Highest increase in the assessed completeness of data reporting was registered in the age group of 13 to 20 years, followed by the group of patients aged 21 to 40 , where $22 \%$ (from $38 \% \pm 11$ to $60 \% \pm 7, p=0.000$ ) and $21 \%$ (from $52 \% \pm 7$ to $73 \% \pm 5$, $p=0.000$ ) more infusions were recorded by the time of e-diary use, respectively. Patients in the age groups of 41 to 60 years (from $74 \% \pm 9$ to $87 \% \pm 4, p=0.001$ ) and $>60$ years (from $80 \% \pm 6$ to $90 \% \pm 3, p=0.002$ ) showed moderate improvement of 13 and $10 \%$, correspondingly, while an improvement of only $2 \%$ (from $65 \% \pm 2$ to $67 \% \pm 2, p=0.05$ ) was seen in the age group of $<13$ years. An overall increase in the completeness of data reporting of $21 \%$ (from $65 \% \pm 35$ to $86 \% \pm 22$, $p=0.003$ ) was registered within the entire cohort.

\section{Assessment of the Impact on Compliance to the Prescribed Treatment Regimen}

Slight overall enhancement of $6 \%$ (from $82 \% \pm 29$ to $88 \% \pm 25, p=0.05$ ) was also assessed in patients' compliance with the prescribed treatment regimen. Major improvement of $24 \%$ was scored in the group of patients between 13 and 20 years, where the parameter showed an increase from $76 \% \pm 10$ to $100 \%, p=0.05$. Only minor improvement (between 2 and 7\%) was observed in the other 4 groups, where $7 \%$ increase was registered in the group of patients older than 60 years (from $92 \% \pm 5$ to $99 \% \pm 2, p=0.05$ ) and $5 \%$ in the group aged 41 to 60 years (from $84 \% \pm 8$ to $89 \% \pm 12, p=0.06$ ). Improvement of $2 \%$ was registered in two of the groups-the one below the age of 13 years and the one between 21 and 40 years (from $98 \% \pm 2$ to $100 \% \pm 0$, $p=0.04$ and from $74 \% \pm 4$ to $76 \% \pm 6, p=0.06$, respectively). However, none of the assessed differences in the parameter proved to be statistically significant.

\section{Assessment of the Impact on Bleeding Episodes Reporting}

Before the introduction of Haemoassist, 32 of 100 patients in the cohort did not report on any bleeding episode. By the time of Haemoassist's use, 16 of those 32 started to report on bleedings $(50 \%$ increase in reporting for the group, $p=0.012$ ). Overview of the whole cohort shows that 68 of 100 patients were reporting by the time of paper diary use, while the number of patients reporting increased to 84 of 100 during the Haemoassist use (16\% increase in the cohort, $p=0.01$ ). Bleeding episode reporting according to patient's age is shown in -Fig. 4.

In summary, significant differences could be assessed for record delivery, completeness of records and bleeding episodes reporting. Borderline level of significance could be assessed for compliance with the prescribed treatment regimen for the whole studied period.

\section{Discussion}

In our analysis, we have studied the impact of the introduction of an e-diary, 'Haemoassist', in patients with severe coagulopathies on the record delivery, the validity of records on compliance to therapy and bleeding events reporting. These parameters were compared with data obtained from the same patients' records collected retrospectively for an antecedent period of paper diary use.

Our study demonstrated an increased number of record deliveries registered in the period of e-diary use in comparison to the period of paper diary use. These findings are consistent with other studies. ${ }^{6,8}$ However, this advantage of Haemoassist use was expected, as the design of the software allows realtime delivery of the entered information to the database system of our treatment centre. In contrast, record deliveries with paper diary used to rely on the patients' visits to the haemophilia care centre. In terms of real-time data transfer, clotting factor tracking as well as therapy follow-up, the ontime data transmission is of utmost importance. ${ }^{13}$ The highest increase in record delivery (34\%) was reported in the age group of 21 to 40 years, which might be related to the convenience of the ubiquitous access to the e-diary via application/webpage, that may be considered as important in the context of presumably active lifestyle of the respective age group. 


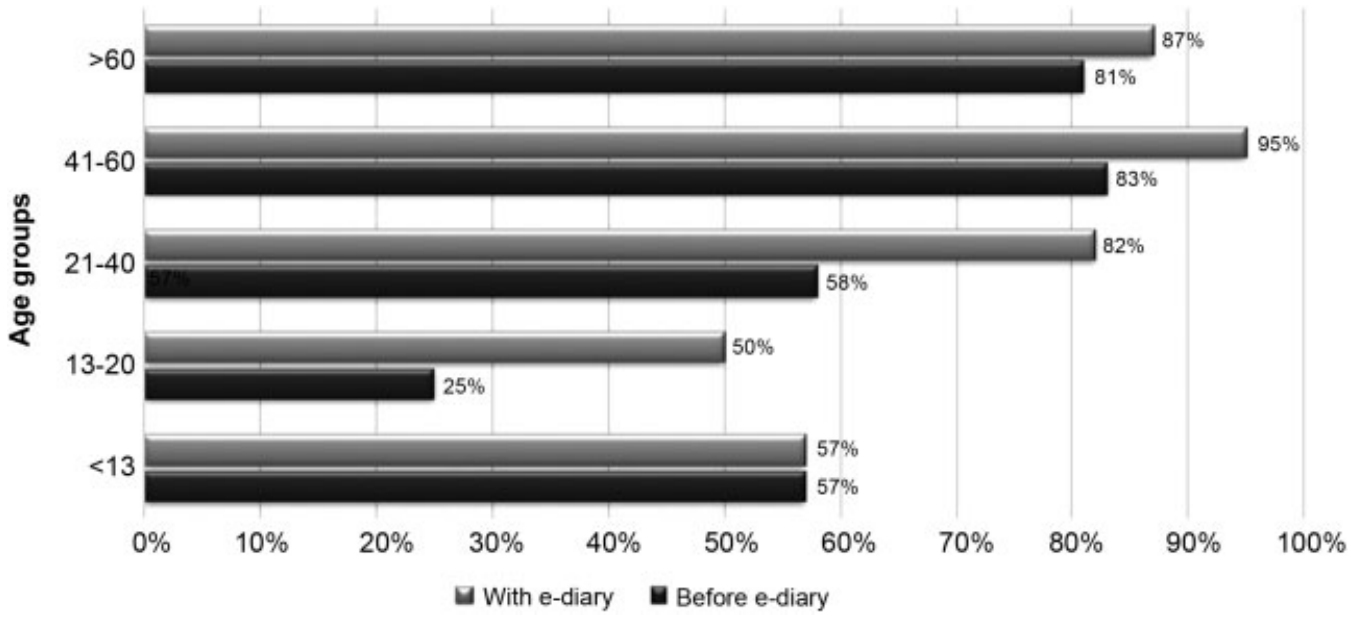

Fig. 4 Age distribution of bleeding episodes reporting for both analysed periods.

Moreover, significant improvements attributed to the use of e-diary were observed in the completeness of data reporting among our cohort. A possible explanation may again be seen in the accessibility of e-diary use. The observed improvements in the completeness of data reporting in the teenage group of patients (13-20 years) and the group of young adults (21-40 years) are deemed as highly relevant, as in our experience therapy tracking in both the age groups is the most cumbersome. Although no or only slight advancement was expected in both the oldest groups (41-60 and over 60 years of age), 10\% increase in the completeness of data reporting was registered, clearly demonstrating that even the older generation of patients is coping with the Internet or application-based diaries and does not consider them demanding.

Furthermore, similar progress was seen in the registration and reporting of bleeding events which again confirmed the suitability and advantages of e-diary use regarding patient's clinical management.

Despite that, the initial compliance with the prescribed treatment regimen in our group of patients proved to be relatively high (73-98\%), improvement in compliance could still be achieved by the switch to e-diary. We could speculate that the calculated difference in compliance of the patients assesses the actual increase in compliance to therapy attributed to the use of 'Haemoassist'. Major progress in the compliance to therapy has been noted in the group of teenagers and young adults, which might be of significance for the joint health preservation in future. ${ }^{14}$ An explanation for the assessed positive effect on the compliance in these two groups may lie in the Haemoassist's reminder function, which assists patients not to forget to apply the prophylactic infusion on the right time and date. To our knowledge, this study was the first to assess the impact of e-diaries on compliance with home treatment in patients with severe bleeding disorders.

Furthermore, the e-diary proves to be an advantageous method for data analysis, as information delivered digitally can be directly integrated into the database of our treatment centre, while the data from paper records must be entered manually into the computer system before processing. ${ }^{5}$ In the past, this process of human transfer of information might have led to entry errors and lack of precision in record transmission, additionally contributing as a confounding factor, generating a deterioration of the assessed results for the evaluated period of paper diary use.

The interpretation of the data is limited by the two different study designs and the different follow-up periods (retrospective data of 3-year period of paper-based diary vs. prospective data of 6-12 months of e-diary use). Furthermore, a part of the results in the prospective arm of the study was obtained by extrapolation, suggesting that the mathematically derived data may differ from the real-life entries and thus could bias the outcome. It also cannot be excluded that certain improvements may be related to the initial attraction of the patients towards the new reporting tool. Nevertheless, the results of this study point to a promising preference of our patients to an electronic transfer of treatment data and the described effects will be re-assessed in the near future with comparable time periods ( 3 years after the introduction of the device) for both documentation tools. Parallel prospective assessment of both record-keeping tools (paper diaries and e-diaries) could be of further benefit to stress the advantages of e-diaries.

\section{Conclusion}

This study aimed to highlight the benefits attributed to the implementation of the e-diary system 'Haemoassist' in haemophilia care. We demonstrated that Haemoassist yields a significant advantage in terms of data recording and tracking of home therapy in haemophilia patients in comparison to paper diaries. Improvement in compliance to prescribed treatment regimen owed to the introduction of the system in the everyday routine of patients and seems to be an additional positive effect.

\section{Conflict of Interest}

J.O. received reimbursement for attending symposia/congresses and/or honoraria for speaking and/or honoraria for consulting and/or funds for research from Bayer, Biogen Idec, Biotest, Chugai, CSL-Behring, Grifols, Novo 
Nordisk, Octapharma, Pfizer, Roche, Shire and Swedish Orphan Biovitrum.

The other authors declare that they have no conflicts of interest.

\section{Acknowledgements}

We would like to express our gratitude to Antonia Dineva, Faculty of Statistics, Technical University Dortmund, Germany, for her assistance with statistical analysis of the data.

\section{References}

1 Teitel JM, Barnard D, Israels S, Lillicrap D, Poon M-C, Sek J. Home management of haemophilia. Haemophilia 2004;10(02):118-133

2 Brackmann HH, Effenberger W, Schwaab R, Hess L, Hanfland P, Oldenburg J. Quality management and quality assurance in haemophilia care: a model at the Bonn haemophilia centre. Haemophilia 2002;8(03):211-216

3 Oldenburg J. Optimal treatment strategies for hemophilia: achievements and limitations of current prophylactic regimens. Blood 2015;125(13):2038-2044

4 Khair K, Holland M. Managing hemophilia : the role of mobile technology. Smart Homecare Technol Telehealth 2014;2:39-44

5 Transfusionsgesetz. Available at: www.juris.de. Accessed February 12,2018
6 Walker I, Sigouin C, Sek J, et al. Comparing hand-held computers and paper diaries for haemophilia home therapy: a randomized trial. Haemophilia 2004;10(06):698-704

7 Baker RI, Laurenson L, Winter M, Pritchard AM. The impact of information technology on haemophilia care. Haemophilia 2004; 10(Suppl 4):41-46

8 Vallée-Smejda S, Hahn M, Aubin N, Rosmus C. Recording practices and satisfaction of hemophiliac patients using two different data entry systems. Comput Inform Nurs 2009;27(06):372-378

9 Mondorf W, Siegmund B, Mahnel R, et al. Haemoassist-a handheld electronic patient diary for haemophilia home care. Haemophilia 2009;15(02):464-472

10 Richert $\mathrm{S}$. Beyond videoconferencing: is your infrastructure ready for telemedicine? Health Manag Technol 2017;38(04):10

11 Langenkamp R. Implementation and migration of haemophiliatreatment-diaries from paper based to electronic documentation at haemophilia CCC at the University of Bonn, Poster Abstract. Res Pract Thromb Haemost 2017;1:1-1451

12 Jamison RN, Raymond SA, Levine JG, Slawsby EA, Nedeljkovic SS, Katz NP. Electronic diaries for monitoring chronic pain: 1-year validation study. Pain 2001;91(03):277-285

13 Sholapur NS, Barty R, Wang G, Almonte T, Heddle NM. A survey of patients with haemophilia to understand how they track product used at home. Haemophilia 2013;19(05):e289-e295

14 Thornburg CD, Duncan NA. Treatment adherence in hemophilia. Patient Prefer Adherence 2017;11:1677-1686 